\title{
TRADUIRE UNE CHANSON DE BRASSENS EN DIALECTE DU SALENTO : DANS L'EAU DE LA CLAIRE FONTAINE
}

\author{
Giulia D'ANDREA \\ Université du Salento
}

\begin{abstract}
Riassunto (It): Il presente articolo si pone l'obiettivo di riflettere su alcune delle problematiche e delle strategie inerenti alla traduzione di una canzone, partendo dall'analisi di una versione inedita in dialetto salentino di Dans l'eau de la claire fontaine di Georges Brassens. La nozione di cantabilità, centrale nel dibattito sulla traduzione cantata, è qui interpretata secondo una prospettiva metrica: stante il carattere sillabico della canzone di partenza e la struttura metrica regolare del suo testo, viene formulata l'ipotesi che la cantabilità di una traduzione cantata possa essere raggiunta attraverso la ricreazione di metro, rima e cadenza.
\end{abstract}

Parole chiave (It): traduzione cantata; traduzione della canzone; cantabilità

Mots-clés (Fr) : traduction chantée ; traduction de la chanson ; chantabilité

DOI : $10.32725 /$ eer.2021.020

\section{Introduction}

Georges Brassens est un des auteurs-compositeurs-interprètes français les plus étudiés du point de vue linguistique, littéraire et stylistique ${ }^{1}$. Son œuvre représente un véritable cas traductologique (CONENNA, 1987; 2000): beaucoup de ses chansons ont été traduites dans les langues les plus variées, du grec à l'allemand, du japonais à l'hébreu et, aussi, dans certains dialectes d'Italie. Pour ces derniers, il suffit de rappeler l'intense activité des interprètes les plus connus, comme Nanni Svampa pour le milanais, Fausto Amodei pour le piémontais, ou Giorgio Ferigo pour le frioulan. Évidemment, l'italien aussi a été souvent choisi comme langue d'arrivée pour ré-interpréter le répertoire de Brassens : parmi les classiques, outre Nanni Svampa, Fabrizio De André a contribué largement à sa diffusion auprès du public italien et Beppe Chierici est le seul traducteur encore vivant à avoir vécu une amitié personnelle avec l'artiste sétois. Parmi les interprètes des générations suivantes, sans viser l'exhaustivité, on peut mentionner Alberto Patrucco, Andrea Belli, Salvo Lo Galbo².

Les artistes susmentionnés ne représentent que la pointe d'un iceberg sous lequel se cache une multitude de traducteurs-amateurs toutes générations et formations confondues qui, sans enregistrer de disques, continuent sans cesse de rendre hommage à l'œuvre immense de Georges Brassens et à la divulguer même dans des cercles privés.

\footnotetext{
${ }^{1}$ À titre d'exemple, v. le numéro double de la revue Équivalences (BRACOPS, 1992) et le volume dirigé par Mirella CONENNA (1998).

${ }_{2}^{2}$ Pour plus d'informations, v. la discographie publiée dans la revue Vinile (DE ANGELIS et alii, 2017).
} 
Dans ce cadre protéiforme et malgré quelques versions dans des dialectes du sud de l'Italie ${ }^{3}$, à notre connaissance, il manquait un travail dans le dialecte du Salento, lacune que nous tentons de combler en relevant le défi de la traduction chantée.

Après avoir illustré le choix du dialecte du Salento comme langue d'arrivée (§1.), et celui de la chanson de départ (§2.), nous passerons aux notes de traduction, qui porteront aussi bien sur des aspects linguistiques et culturels que sur la notion de chantabilité (§§3.-5.); pour finir, nous évoquerons le rôle des traductions existantes (§6.).

\section{La langue d'arrivée : le dialecte du Salento}

Parlé dans la partie méridionale des Pouilles (Italie), ce dialecte est une variété romane caractérisée par un système à cinq voyelles (GRASSI, SOBRERO, TELMON, 1997 : 93-94), qui se différencie du système vocalique de l'italien standard par le manque des oppositions phonématiques e ouvert vs e fermé, ainsi que o ouvert vs $o$ fermé. Parmi les traits consonantiques prégnants, signalons les sons rétroflexes ou cacuminaux, qui s'articulent avec la langue courbée en arrière (ROHLFS, 1966 : 328).

Les voyelles atones en syllabe finale ne subissent pas d'affaiblissement ni de chute, mais elles sont toujours conservées. En l'occurrence, $a$ est toujours maintenu dans la presqu'île du Salento (RoHLFS, 1966 : 176) ; dans la province de Lecce, le $e$ et le $i$ atones en syllabe finale sont différenciés (ROHLFS, $1966: 183$ ); à la place du $o$ et du $u$ du toscan, dans le Salento, on trouve généralement $u$, sauf à la troisième personne du pluriel de certains temps verbaux (ROHLFS, 1966 : 187-188).

Comme nous le verrons, cette précision concernant les voyelles atones en syllabe finale n'est pas sans conséquences pour la traduction chantée, car la nonchute des voyelles finales en position posttonique contribue à accentuer l'un des problèmes majeurs, à savoir la re-création des vers oxytons du français. En revanche, cette difficulté, qui concerne aussi la langue italienne, est atténuée dans certains dialectes gallo-romans ${ }^{4}$.

Pour notre traduction chantée, nous avons adopté le dialecte parlé notamment dans l'aire de Lecce, car il fait partie de notre répertoire linguistique personnel. Dans notre métalangage, vu l'optique traductologique adoptée ici, nous y renvoyons par l'expression langue d'arrivée, qui insiste sur la fonction de ce code au sein de l'opération traduisante, indépendamment des considérations liées au statut de prestige dont il peut jouir.

\footnotetext{
${ }^{3}$ Que l'on songe aux versions en sicilien réalisées par Salvatore Pagano. La Supplique pour être enterré à la plage de Sète a été traduite en dialecte de Bari par Vito Carofiglio en 1991 et chantée sur scène par Rocco Capri Chiumarulo, lors de la journée d'étude Tradurre in musica. Omaggio a Brassens (Université de Bari, 14 mars 2005). À l'occasion du Premio Brassens organisé en 2019 par la Mairie de Marsico Nuovo en Basilicate, Michele Casale a interprété U sciavuórte, traduction-relais du Gorille réalisée en 1997 par Adriano Cozza à partir du texte pivot de Fabrizio De André.

${ }^{4}$ Sur ce point, v. aussi D'ANDREA (2014).
} 


\section{La chanson de départ}

Dans l'eau de la claire fontaine a été publiée en 1961 dans l'album Trompettes de la renommée. À la différence de certaines chansons de Brassens, situées dans un contexte urbain typiquement parisien et qui ne peuvent pas s'exporter facilement sans renoncer à leur couleur locale (ZOPPI, $1998: 16$ ), cette chanson se prête bien à être re-située ailleurs car elle évoque une scène dépourvue de toute référence spatiotemporelle :

Dans l'eau de la claire fontaine

Elle se baignait toute nue.

Une saute de vent soudaine

Jeta ses habits dans les nues.

En détresse, elle me fit signe,

Pour la vêtir, d'aller chercher

Des monceaux de feuilles de vigne,

Fleurs de lis ou fleurs d'oranger.

Avec des pétales de rose,

Un bout de corsage lui fis.

La belle n'était pas bien grosse:

Une seule rose a suffi.

Avec le pampre de la vigne,

Un bout de cotillon lui fis.

Mais la belle était si petite

Qu'une seule feuille a suffi.

Ell' me tendit ses bras, ses lèvres,

Comme pour me remercier...

Je les pris avec tant de fièvre

Qu'ell' fut toute déshabillée.

Le jeu dut plaire à l'ingénue,

Car à la fontaine, souvent,

Ell' s'alla baigner toute nue

En priant Dieu qu'il fìt du vent,

$Q u$ 'il fit $d u$ vent...

La chanson raconte l'histoire d'une rencontre entre le canteur - terme qui indique « l'équivalent du narrateur dans un roman » (HIRSCHI, $2008: 20$, n. 4) - et une jolie fille ${ }^{6}$.

\footnotetext{
${ }^{5}$ Les paroles sont tirées de BRASSENS $(1973: 145)$. Au v. 9, nous signalons la variante « roses » pour « rose », attestée dans SvAMPA, MASCIOLI (1991: 144), un ouvrage devenu incontournable pour les traducteurs italiens de Brassens.

${ }^{6}$ Celle-ci est en train de se baigner toute nue auprès d'une source, la « claire fontaine » (v. 1), lorsque ses vêtements sont soudainement emportés par le vent. Empêchée de se revêtir, elle appelle en secours le canteur, qui, jusqu'à ce moment, a assisté à la scène sans proférer mot. Par des gestes, elle lui demande « d'aller chercher des monceaux de feuilles de vigne, fleurs de lis ou fleurs d'oranger » (v. 68). Une seule rose et une seule feuille de vigne suffisent pour réaliser un cotillon et un corsage pour la jeune fille ; celle-ci cherche à le remercier en lui tendant ses bras et ses lèvres mais, puisque le canteur
}

$$
-111 \text { - }
$$


L'intrigue narrative se développe selon des séquences qui reposent sur un dialogue muet fait de gestes ( « elle me fit signe», v. 5 ; « un bout de corsage/cotillon lui fis », v. 10 et 14 ; « ell' me tendit ses bras, ses lèvres », v. 17 ; « je la pris avec tant de fièvre », v. 19). La protagoniste est entourée par un halo d'indéfini : l'auteur n’y renvoie que par un générique « elle » (v. 2, 5, 17, 20, 23), par les pronoms compléments d'objet direct «la» (v. 6), et indirect «lui» (v. 10, 14); les seules qualités qui lui soient explicitement attribuées sont la beauté ( «la belle n'était pas bien grosse », v. 11 ; «mais la belle était si petite», v. 15) et l'ingénuité (« le jeu dut plaire à l'ingénue », v. 21). Dans la description, elle n'a pas d'âge précis, mais on peut deviner sa jeunesse par des indices : sa virginité est suggérée par le «lis », symbole de la pureté, et par les «fleurs d'oranger » (v. 8), qui évoquent une mariée d'antan, censée arriver vierge au mariage ; les feuilles de vignes, autrefois utilisées pour cacher les nudités des statues romaines, symbolisent la pudeur; les roses sont des métaphores notoires de la beauté, de la jeunesse et de l'amour. Néanmoins, cette ingénuité n'est pas tout à fait cohérente avec l'image d'une fille qui s'expose à des regards indiscrets en se baignant « toute nue » (v. 23) et, surtout, qui revient souvent au même endroit « en priant Dieu » (v. 24) pour que l'histoire puisse recommencer à nouveau.

Les indices linguistiques qui renvoient au canteur sont des embrayeurs tels le pronom complément de première personne du singulier «me », direct (v. 18) ou indirect (v. 5, 17), et le pronom personnel sujet «je», exprimé (v. 19) ou sousentendu (v. 10,14). La première personne du singulier est également implicite dans deux propositions subordonnées infinitives dont la première dépend de la seconde (v. 6). Le portrait qui en ressort est d'abord celui d'un œil qui assiste passivement à la scène discrètement érotique de la baignade sans voiles (strophe 1), ensuite celui de quelqu'un qui n'intervient que pour répondre aux souhaits de la jeune fille (strophes 2, 3 et 4) et, enfin, celui d'un homme qui exprime son ardeur (v. 19). Ce crescendo de séduction et d'ardeur est brusquement interrompu par le retour à la situation initiale de nudité de la fille (v. 20). La dernière strophe, pour boucler la boucle, fait écho à la première par l'absence de tout indice linguistique renvoyant au canteur, qui revient dans l'ombre.

Cet aperçu du texte de la chanson permet de formuler quelques réflexions concernant sa traduction, et notamment d'identifier les éléments que nous avons jugés essentiels pour la récréer dans un autre code linguistique sans que n'en soient trahis ni la lettre ni l'esprit. Premièrement, la scène se déroule dans un temps indéfini; deuxièmement, le contexte est marqué par une source d'eau; troisièmement, le personnage féminin est décrit dans son apparente naïveté qui cache en réalité une malice discrète.

Tout en sachant qu'une traduction comporte des pertes, nous veillerons à sauvegarder des aspects incontournables du texte de départ, tels que le cadre atemporel, le paysage aquatique et l'ambiguïté du personnage féminin. Quant à la structure formelle, nous essayerons de respecter le schéma métrique et rimique de

l'étreint ardemment, elle reste à nouveau sans vêtements. Elle se laisse séduire par la situation au point qu'elle aimera revenir souvent au même endroit, en espérant que ce jeu de malice discrète ne s'arrêtera jamais. 
l'original. Dans les paragraphes 4 et 5 , nous montrerons de quelle manière nous avons atteint ces objectifs.

\title{
3. Le texte d'arrivée : avertissement
}

La difficulté de bien des dialectes est la manière de les orthographier; nous présentons cependant ci-dessous une tentative de transcription du texte d'arrivée. Faute d'un système de graphie univoque, les caractères utilisés sont ceux de la langue italienne.

Pour indiquer les consonnes rétroflexes sonores issues du groupe latin -LL-, nous avons adopté le trigramme $d d h$ (bbeddha, $d d h a$ ), selon une des conventions les plus utilisées dans la littérature dialectale. Quant aux consonnes rétroflexes sourdes, nous ne les avons pas signalées de manière explicite, mais il faut bien préciser qu'une prononciation cacuminale concerne aussi bien le groupe $\operatorname{tr}$ (ttre, quattru) que le groupe str (stria). Du côté de la prononciation, une fricative post-alvéolaire sourde correspond aussi bien au digramme sc (sciuta, scecu) qu'à la consonne $c$ suivie d'une voyelle palatale (piacia, face). Quant à la consonne $s$ en position préconsonantique, elle est aussi prononcée comme une fricative post-alvéolaire sourde [J] dans le groupe /st/ (stia, esta, estere, bbastau), mais non pas dans les groupes $/ \mathrm{sp} /$ et $/ \mathrm{sk} /$, où elle garde une articulation alvéolaire (spicciau, scupa, vespa) ${ }^{7}$. Pour une meilleure lisibilité de la part d'un lecteur non dialectophone, nous avons marqué graphiquement le redoublement phonosyntaxique (cu nni cсоnzu).

\author{
Ia sciuta sse face lu bbagnu \\ Ammeru alla grotta Poesia \\ Stia nuta finc 'allu carcagnu \\ Cce beddha ca era ddha stria \\ Spicciau lu faugnu e llu ientu \\ All'aria la esta menau \\ Ddha fiata foi mutu cuntentu \\ Ca la bbeddha stria me chiamau \\ Ulia cu nni acchiu ddo fiuri \\ Cu sse pozza estere ma \\ La stria era nna mazza te scupa \\ E nnu fiuru sulu bbastau \\ Ulia cu nni cconzu nna esta \\ Cu tre quattru pampane ma \\ Tenia nnu vitinu te vespa \\ E nna fogghia sula bbastau \\ Me fice ddha stria l'ecchiu rizzu \\ Li musi e lle razze ntesau \\ E comu ncignai lla ncarizzu \\ La stria tutta nuta restau
}

\footnotetext{
7 La prononciation du groupe str mériterait une étude à part en raison du phénomène de cacuminalisation (v. ROHLFS, $1966: 259$ ). 
M'a parsa nna capicalata

Ddhu scecu ormai ni piacia

Te tandu ogne ggiurnu a tturnata

Ammeru alla grotta Poesia

Grotta Poesia

Pour que le texte d'arrivée puisse être reçu par un public francophone qui n'est pas censé connaître le dialecte du Salento, nous l'avons re-traduit vers le français. Ce passage ultérieur ayant pour but de faciliter le décodage du texte dialectal et la compréhension des choix opérés lors de la traduction chantée, nous avons appliqué les principes du mot-à-mot et du vers-à-vers. Le critère de la littéralité, facilité par l'origine romane commune du français et du dialecte du Salento, a été adopté jusqu'au respect de l'ordre des mots, sauf s'il constitue une entrave à la compréhension en français :

$<$ Elle $>$ était allée se baigner

Auprès de la grotte $<$ de la $>$ Poésie

$<$ Elle $>$ était nue jusqu'aux pieds

Qu'elle était belle, cette fille!

La touffeur cessa et le vent

En l'air sa robe emporta

Alors je fus très content

Que la jolie fille m'appelât

$<$ Elle $>$ voulait que je lui cherche des fleurs

Pour qu'elle puisse s'habiller mais

La fille était un manche à balai

Et une fleur seule suffit

$<$ Elle $>$ voulait que je lui apprête une robe

Avec trois ou quatre pampres mais

$<$ Elle $>$ avait une taille de guêpe

Et une feuille seule suffit

Cette fille me fit un clin d'œil

Les lèvres et les bras $<$ me $>$ tendit

Et dès que je commençai à la caresser

La fille toute nue resta

$<$ Elle $>$ avait l'air de jouer les ingénues

Ce jeu désormais lui plaisait

Dès lors chaque jour $<$ elle $>$ est revenue

Auprès de la grotte $<$ de la $>$ Poésie

Grotte $<$ de la $>$ Poésie

\section{Chanter «presque la même chose »}

Pour qu'on puisse parler de traduction et non pas d'adaptation, notre version dialectale de la chanson de Brassens respecte le thème de la chanson originale, depuis l'histoire racontée jusqu'au décor naturel, caractérisé par la présence de l'eau 
et du vent. Néanmoins, des pertes et des ajouts ont été nécessaires pour des raisons de traductibilité et de chantabilité.

À l'encontre du texte de Brassens, qui décrit un cadre dépourvu de toute référence géographique, nous avons choisi de donner une localisation précise à la version en dialecte du Salento, à savoir la grotte de la Poésie («grotta Poesia », v. 2), un endroit connu dans la région, situé le long de la côte adriatique et célèbre pour la clarté de ses eaux. Dans l'imaginaire collectif, cette localité est entourée d'un halo mythique, riche de légendes comme celle d'une belle princesse qui aimait s'y baigner en attirant ainsi des poètes et des écrivains, d'où le nom de grotta della Poesia, que nous avons abrégé en " grotta Poesia » pour des raisons de chantabilité. Au-delà de l'étymologie plus ou moins fantaisiste, l'ajout du toponyme a pour effet de rendre la version dialectale plus crédible, et d'en faciliter la réception par le public du Salento.

Le même objectif est visé par un choix de surtraduction concernant la protagoniste de l'histoire : là où le texte français n'y renvoie que par les pronoms personnels de troisième personne, nous avons opté pour une solution plus explicite. Nous avons donc adopté le substantif stria ('fille'), qui détermine approximativement son âge. Pour ce qui concerne sa description, nous avons traduit l'adjectif ingénue par le substantif capicalata ('sainte nitouche'), qui rend parfaitement l'idée de la fausse naïveté et de la malice voilée de la fille, idée sousjacente au texte entier. Par une sorte de compensation, l'audace de ce choix traductif est estompée par le contexte phrastique, qui exprime la subjectivité de l'opinion: « m'a parsa nna capicalata» (v. 21, 'j'ai eu l'impression qu'elle était une sainte nitouche'). Comme c'est souvent le cas en traduction, on interprète : tantôt en accentuant, tantôt en atténuant un aspect du texte original. Dans l'exemple suivant, la compensation se cache derrière un cas apparent de sous-traduction concernant les vers 5-6 :

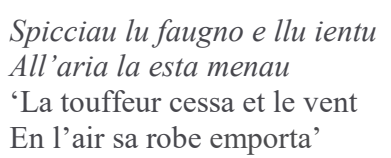

sémantiquement équivalents aux vers 3-4 du texte de départ ${ }^{8}$ :

Une saute de vent soudaine

Jeta ses habits dans les nues.

Ici, les connotations portées par saute et soudaine, apparemment omises dans la traduction, sont en réalité récupérées grâce à l'ajout de « spicciau lu faugnu » (v. 5). En effet, la coordination des deux propositions « Spicciau lu faugnu » et « 1lu ientu all'aria la esta menau » permet à l'auditeur d'imaginer très clairement la sensation libératrice que l'on éprouve dans le Salento lorsqu'un peu de vent se lève après d'interminables journées caractérisées par l'absence de vent et par ce qu'on appelle

\footnotetext{
${ }^{8}$ Nous profitons de cet exemple pour préciser qu'une traduction chantée vers à vers serait sinon impossible, du moins très difficile à atteindre.
} 
faugno 9 , terme qui contribue à créer une certaine couleur locale et à accentuer la crédibilité de notre traduction.

Le même effet est obtenu par deux autres choix traductifs consistant à utiliser des phrases figées à la place de phrases libres :

\section{La belle n'était pas bien grosse (v. 11)}

Mais la belle était si petite (v. 15)

Ces deux vers concernent l'aspect physique de la jeune fille, en insistant respectivement sur sa minceur (v. 11) et sur sa taille ou sur son âge (v. 15). Ayant déjà explicité la jeunesse de la figure féminine par le substantif stria ('fille'), nous avons traduit ces deux vers en privilégiant la description de sa minceur, trait pour lequel une seule fleur et une seule feuille suffisent pour couvrir son corps. Pour exprimer l'idée de la minceur, nous avons opté pour deux expressions figées qui, par leur caractère imagé, font en sorte que les auditeurs en soient frappés et qu'ils puissent visualiser la scène décrite avec plus d'efficacité. Voici la traduction du v. 11 :

\section{La stria era nna mazza te scupa}

'La fille était un manche à balai'

Ce vers, contenant le nom composé mazza te scupa pris au figuré, pourrait être paraphrasé par 'la fille était très mince', ce qui annulerait toute connotation négative associée à la locution dialectale. Dans la glose, nous avons donc préféré adopter la locution française manche à balai qui possède, elle aussi, une certaine connotation négative. Nous sommes néanmoins consciente que deux expressions figées dans deux langues différentes peuvent ne pas obéir aux mêmes conditions d'emploi malgré l'analogie lexicale, structurelle et sémantique (CAMUGLI GALLARDO, 2010).

Passons maintenant à la traduction du v. 15 :

Tenia nnu vitinu te vespa

' $<$ Elle $>$ avait une taille de guêpe'

Dans ce cas, l'expression vitinu te vespa peut être considérée comme une forme de compensation car, en plus de la minceur, elle véhicule une connotation positive et renvoie à une beauté attrayante. Cette expression existe en italien aussi (vita o vitino di vespa), où elle serait un calque du français (GDLI, s.v. vespa, vol. XXI : 811).

Les exemples illustrés révèlent que nous avons traduit en "cibliste" (LADMIRAL, 2015 : 20), car les paroles de la chanson d'arrivée semblent avoir été conçues directement en dialecte. Mais si une chanson traduite accroche les auditeurs

\footnotetext{
${ }^{9}$ Le mot faugnu, comme le français foehn, est issu du latin classique favonius. Cependant, ces deux mots ont deux significations différentes : alors que foehn indique un vent sec et chaud, faugno renvoie plutôt à une chaleur humide. Voilà pourquoi, dans notre glose, nous l'avons traduit par touffeur.
} 
comme s'il s'agissait d'un original, c'est qu'elle obéit aussi au principe de la chantabilité.

\section{Une traduction « à chanter»}

Lorsqu'on traduit un texte destiné au chant, on a essentiellement deux options traductives : réaliser une traduction à lire du texte en question, qui ne tienne pas compte de la dimension musicale, ou réaliser une traduction à chanter (D'ANDREA, 020). Dans ce dernier cas, la musique joue un rôle majeur, car elle influe sur les choix du traducteur et qu'en même temps, elle lui permet d'atteindre une certaine fidélité à la chanson de départ par le biais de la chantabilité, critère dont on arrive à saisir la signification de manière intuitive malgré l'absence d'une définition universellement partagée.

Nous postulons ici que pour établir une traduction chantable d'une chanson de Brassens, il est grandement utile de respecter la composante métrique de son texte ; et ceci malgré l'existence de certaines traductions à succès qui n'obéissent pas à ce critère comme, par exemple, celles de Fabrizio De André. L'équivalence métrique des paroles de chanson, en somme, serait une condition suffisante mais pas nécessaire pour qu'une chanson traduite soit chantable ${ }^{10}$. Notre hypothèse se fonde sur le double constat que dans les chansons de Brassens, chaque syllabe du texte est généralement chantée sur une seule note et que les paroles de ses chansons ne sont pas seulement formatées selon les critères de la poésie écrite mais aussi caractérisées par une certaine régularité métrique. Celle-ci découle d'une profonde connaissance de la poésie classique de la part de l'auteur, tout en présentant quelques caractéristiques propres à la poésie de tradition orale ${ }^{11}$.

Or, les paroles de Dans l'eau de la claire fontaine sont articulées en six quatrains d'octosyllabes à cadences alternées (féminine et masculine) et contiennent aussi bien des assonances que des contre-équivalences, deux procédés typiques de la tradition orale (CORNULIER, 2004) ${ }^{12}$.

Nous montrerons de quelle manière la version dialectale de cette chanson cherche à faire revivre la chanson de Brassens en passant aussi par une récupération des aspects formels évoqués ci-dessus; pour plus de clarté, notre discours sera divisé en quatre parties portant respectivement sur le mètre (\$5.1.), la cadence (§5.2.), la rime (§5.3.) et la contre-équivalence (§5.4.).

\subsection{Le mètre}

Indépendamment de leur mise en musique, les paroles d'une chanson peuvent être analysées comme s'il s'agissait de poésie de tradition littéraire. Néanmoins, même chez Georges Brassens, notoirement féru de poésie classique, la scansion des unités traditionnellement appelées vers ne se fonde pas uniquement sur des critères

\footnotetext{
${ }^{10}$ Cette affirmation est en réalité une simplification car il est également important de respecter un certain agencement de syllabes accentuées et non accentuées.

${ }^{11}$ Pour une analyse systématique de la métrique des textes de toutes les chansons de Georges Brassens, v. ACHiLle (2007).

12 Rappelons au passage que par le syntagme la claire fontaine, Brassens fait écho à la chanson traditionnelle $\dot{A}$ la claire fontaine.
} 
propres à la poésie écrite mais dépend également de la manière dont ces vers sont chantés sur la musique. Il suffit de penser au -e instable à la fin des mots, dont la réalisation optionnelle dans le chant a des implications sur le nombre de syllabes pertinent pour réaliser une traduction métriquement fidèle. Il est particulièrement intéressant de noter que cette double option arrive jusqu'à concerner le même mot dans la même chanson. Pour donner un exemple tiré de Dans l'eau de la claire fontaine, le pronom personnel elle est traité de deux manières différentes dans le chant, en fonction du nombre de notes auxquelles correspondent ses deux syllabes graphiques (el-le) : si celles-ci tombent chacune sur une note, le mot est scandé en deux syllabes métriques (v. 2 et v. 5); si, au contraire, le mot perd sa deuxième syllabe dans sa réalisation chantée, il sera scandé en une seule syllabe (v. 17 et v. 23). Chez Brassens, ce second traitement est même transcrit sur le papier (ell'), ce qui n'est pas toujours le cas pour les paroles de chanson des autres auteurscompositeurs-interprètes.

Pour établir le mètre des vers de départ de notre chanson, nous avons calculé leur longueur anatonique $\left(\mathrm{L}_{\mathrm{a}}\right)$, à savoir le nombre de voyelles métriques jusqu'à la dernière voyelle tonique y compris celle-ci (CORNULIER, 1999, s.v. anatonique). Puisque $\mathrm{L}_{\mathrm{a}}=8$, nous pouvons affirmer qu'il s'agit de vers octosyllabes, à savoir de vers dont la dernière voyelle tonique se trouve en huitième position. D'après les conventions de la métrique italienne, un vers équivalent serait appelé novenario, car les vers italiens sont dénommés sur la base des vers paroxytons et non sur la base des vers oxytons. Or, la terminologie de Cornulier, que nous appliquons à l'italien et ici au dialecte du Salento, a l'avantage de neutraliser les différences dans la dénomination des vers français et italiens et de faciliter ainsi la comparaison entre le système métrique de la langue française et celui du dialecte du Salento.

Dans cette optique, notre texte d'arrivée se compose de six quatrains de vers ayant une longueur anatonique $=8$. Pour identifier les voyelles métriques des vers d'arrivée, il faut tenir compte des figures métriques qui permettent de fusionner deux ou plusieurs voyelles contiguës dans une seule syllabe ou, au contraire, de les séparer. Dans la versification italienne, ainsi que dans la poésie dialectale du Salento ${ }^{13}$, ces figures métriques peuvent se trouver aussi bien à l'intérieur d'un mot qu'à la frontière entre deux ou trois mots.

Lorsque les voyelles contiguës se trouvent à l'intérieur d'un mot, ces figures métriques sont respectivement appelées synérèse et diérèse. Lorsque les voyelles se trouvent à la frontière des mots, les figures prennent le nom de synalèphe et de dialèphe $^{14}$. Par exemple, nous avons adopté une synérèse dans $u l i \underline{a}^{15}$ [u.lia] (v. 9), une diérèse dans faugnu [fa.u.nu] (v. 5) et une synalèphe dans la suite ammeru alla [a.m:c.rua.1:a] (v. 2). Nous n'avons pas adopté de dialèphe.

\footnotetext{
${ }^{13}$ Pour la poésie dialectale, nous avons vérifié le bien-fondé de cette affirmation dans le corpus de poèmes composés par Francesc'Antonio D’Amelio (1775-1861) et Francesco Marangi (1864-1939), publiés dans VALLI (1998).

${ }^{14}$ Nous traduisons ici par des calques la terminologie de la versification italienne, tout en sachant que dans la tradition métrique française, ces quatre phénomènes ont une distribution différente, et qui varie aussi au fil des siècles.

${ }^{15}$ Nous soulignons ici les graphèmes correspondant aux voyelles concernées par la figure métrique en question.
} 
Quant à l'agencement des syllabes accentuées et inaccentuées, les paroles du texte de départ respectent les contraintes de la métrique littéraire : la dernière voyelle tonique de chaque vers se trouve en huitième position, tandis que les accents intérieurs observent une certaine mobilité. La variabilité rythmique qui en découle est même plus grande dans la version enregistrée par Georges Brassens, avec les pauses et les accélérations propres à l'interprétation vocale. Dans notre traduction chantée, nous avons volontairement créé un schéma plus régulier, en plaçant les voyelles porteuses d'accent presque toujours sur les mêmes positions $\left(2^{\mathrm{e}}, 5^{\mathrm{e}}\right.$ et $\left.8^{\mathrm{e}}\right)$, afin de faciliter une interprétation chantée de cette ballade dans le style des chansons populaires du Salento.

\subsection{La cadence}

Si nous avons pu reconstruire en langue d'arrivée le mètre des vers de départ, il n'en a pas été ainsi pour ce qui concerne leur cadence, le deuxième paramètre retenu pour décrire la métrique des paroles d'une chanson. Le schéma de la chanson de Brassens se fonde sur une parfaite alternance de vers à cadence féminine et de vers à cadence masculine, qui fait pendant à la structure mélodique de la chanson. Pour le dire avec les termes de CORNULIER (1999, s.v. cadence), ce sont des vers dont la longueur catatonique $\left(\mathrm{L}_{\mathrm{c}}\right)$, à savoir le nombre de voyelles métriques qui vont de la dernière voyelle tonique jusqu'à la fin du vers, est respectivement $=2$ et $=1$. Or, il est bien connu que les caractéristiques prosodiques de la langue italienne rendent assez difficile la création de vers ayant une $\mathrm{L}_{\mathrm{c}}=1$, faute d'un nombre important de mots oxytons. Voilà pourquoi, en traduction chantée vers l'italien, on opte souvent pour des stratagèmes, telle l'adoption de monosyllabes ou de mots proparoxytons, qui contribuent à créer une certaine variété et à atténuer l'effet de monotonie résultant d'un emploi massif de certaines personnes verbales du futur simple et du passé simple.

Comme nous l'avons montré précédemment (§1.), dans le dialecte du Salento les voyelles atones en syllabe finale ne sont ni amuïes ni affaiblies. Ce qui implique un nombre très limité de mots oxytons. Pour essayer de respecter les cadences masculines de la chanson de départ dans la chanson d'arrivée, nous avons donc eu recours à des stratégies métriques. Outre le monosyllabe $m a$, nous avons adopté des mots se terminant par un digramme, comme les noms poesia et stria ou des verbes à la troisième personne de l'imparfait (piacia) ou du passé simple (menau, chiamau, bbastau, ntesau, restau). Dans la poésie dialectale de tradition littéraire, ces mots sont généralement traités en synérèse à l'intérieur des vers ; au contraire, ils sont traités comme des mots paroxytons en fin de vers ${ }^{16}$. En revanche, la mise en chanson de ces mots élargit la palette des possibilités : lorsque les éléments vocaliques des digrammes sont associés à deux notes distinctes, on aura l'impression d'un traitement en diérèse; si, par contre, chaque digramme est associé à une seule note dans le chant, il sera perçu comme rythmé en synérèse.

\footnotetext{
${ }^{16}$ Cette affirmation se fonde sur le dépouillement d'un corpus de textes poétiques en dialecte de Lecce contenus dans VALLI (1998). De même, dans la tradition poétique italienne, les groupes de voyelle tonique + voyelle atone en fin de mot comptent pour deux syllabes à la fin du vers, alors qu'à l'intérieur d'un vers ils comptent pour une syllabe (BELTRAMI, $1996: 35$ ).
} 
Ainsi, dans notre chanson d'arrivée, les deux voyelles contiguës des digrammes $-i a$ et - $a u$ sont chantées sur une seule note même lorsqu'elles sont en fin de vers, ce qui représente une sorte de licence poétique du chant par rapport à la métrique du texte écrit. À l'écoute, on a ainsi l'impression que l'alternance de vers féminins et masculins de la chanson de Brassens a été recréée dans la chanson d'arrivée.

\subsection{La rime}

La rime est ici considérée comme le troisième paramètre métrique susceptible de jouer un rôle dans la mise au point d'une traduction chantée. Dans les paroles de la chanson de départ, la rime relie de manière systématique des constituants métriques relevant de deux niveaux distincts : les vers et les modules. En effet, prendre en compte le terme rime dans un sens large englobant aussi les assonances ou rimes vocaliques « rose »/《 grosse » (v. 9 et 11) et « vigne »/《petite» (v. 13 et 15) équivaut à affirmer que les vers de Dans l'eau de la claire fontaine sont en rimes croisées. En revanche, si l'on s'en tient à une définition stricte de rime ${ }^{17}$, qui exclut l'assonance, la périodicité rimique se manifeste à un niveau autre que celui des vers, niveau appelé module. Par ce terme, CORNULIER (1999, s.v. module) indique tout « constituant strophique de niveau supérieur au vers, pouvant entrer en relation rimique simple ou composée avec d'autres modules ». Les paroles de notre chanson de départ seraient donc articulées en quatrains rimés en (aa).

Lors du processus traductif, non seulement nous avons considéré la rime comme un paramètre non accessoire, mais nous l'avons parfois même érigée en principe fondamental dans la création du texte d'arrivée. Par exemple, dans la $5^{\mathrm{e}}$ strophe, c'est grâce à la contrainte de la rime avec ncarizzu (dont la forme catatonique est /its:u/) que nous avons trouvé la solution créative consistant à rendre le geste de la fille («Elle me tendit ses bras, ses lèvres », v. 17) par un clin d'œil (l'ecchiu rizzu, rimant justement avec ncarizzu). Ailleurs, nous nous sommes contentée d'une assonance, à savoir d'une « forme peu exigeante de la rime » (CORNULIER, 1999, s.v. rime) : dans ce cas, l'équivalence entre deux unités métriques se limite aux seules voyelles de leurs formes catatoniques. Ainsi, dans la $4^{\mathrm{e}}$ strophe, les formes catatoniques d'esta ${ }^{18}$ et de vespa (/عfta/ et /عspa/) n'ont en commun que les sons vocaliques.

Dans la tentative de recréer le schéma rimique de la chanson originale dans la chanson d'arrivée, nous avons essayé de tenir compte aussi bien du niveau des vers que du niveau des modules, avec ceci comme résultat : le schéma de rimes croisées entre les vers n'est complet que dans les strophes 1,2, 5 et 6 ; quant aux strophes 3 et 4 , celles du texte d'arrivée prennent plus de liberté par rapport à la chanson de

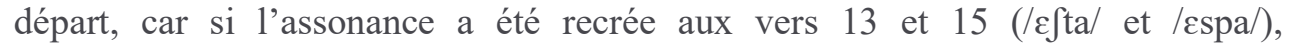
l'équivalence de forme catatonique entre les vers 9 et 11 est réduite à la seule dernière voyelle masculine /u/. En outre, la rime de module n'est pas respectée car

\footnotetext{
${ }^{17}$ Nous rappelons au passage que d'après CORNULIER (1999, s.v. rime) il y a rime intégrale entre deux expressions métriques en présence d'une équivalence phonématique complète de leurs formes catatoniques, à savoir de leurs parties non-prétoniques.

${ }^{18}$ Ce mot a été choisi comme variante phonétique d'este pour des raisons de rime. -120 -
} 
la séquence catatonique des vers 12 et 16 contient une semi-voyelle de plus par rapport à celle des vers 10 et 14 .

\subsection{La contre-équivalence}

Les strophes centrales de la chanson de départ (3 et 4), tout en étant moins rigoureuses sur le plan du schéma rimique, présentent des caractéristiques assez spécifiques pour ce qui concerne la contre-équivalence. En forgeant ce terme, CORNULIER (2004) vise à considérer certaines formes de répétition comme un fond d'équivalence pour la mise en évidence de contrastes qui peuvent se situer à des niveaux de langage différents. Dans le cas qui nous intéresse, des parties de vers se répètent d'une strophe à l'autre, ce que nous indiquons en italique :

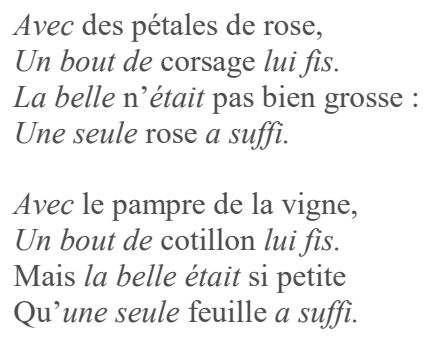

Or, ces équivalences discontinues d'une strophe à l'autre représentent notamment «le support fonctionnel» (CORNULIER, 2004: 6) des contrastes lexicaux entre les paires de mots suivants : corsage/cotillon; rose/feuille. En réalité, du point de vue phonétique, l'équivalence entre les vers 2 des deux strophes arrive jusqu'à comprendre la consonne initiale des deux mots en contraste, le / $/ \mathrm{k} / \mathrm{de}$ corsage et de cotillon $^{19}$. En traduisant, nous avons tenu compte de ces éléments et nous avons recréé le réseau d'équivalences et de contrastes de la manière suivante :

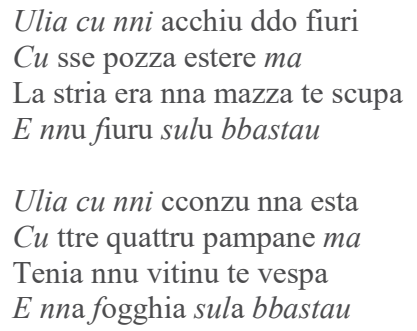

Les répétitions indiquées en italique ont pour but de mettre en évidence des contrastes sur le plan lexical, comme celui entre les expressions cconzu nna esta et acchiu ddo fiuri et celui entre les mots fiuru et fogghia. La différence de genre entre ces deux noms implique aussi un contraste phonétique entre les déterminants ( $n n \mathrm{u} /$ nna) et entre les adjectifs épithètes (sulu / sula). Nous avons eu recours aux caractères droits et italiques pour indiquer les graphèmes qui représentent des sons.

\footnotetext{
${ }^{19}$ Une première ébauche de cette analyse a été réalisée en collaboration avec Elide Achille et présentée à la journée d'études Tradurre in musica. Omaggio a Brassens (Université de Bari, 14 mars 2005). 
Néanmoins, dans certains cas la stratification des niveaux est plus complexe que cela car derrière une répétition lexicale et phonétique comme celle de $c u$ au début du vers 2 , se cache en réalité un contraste morpho-syntaxique : en effet, le premier $c u$ est une conjonction de subordination à valeur finale ('pour que') et le deuxième $c u$ est une préposition ('avec').

\section{L'apport des traductions existantes}

Une fois la traduction achevée, nous avons observé que certains choix traductifs présentent des analogies avec des versions italiennes de la même chanson. Ce qui s'explique par le fait qu'un traducteur de chansons est avant tout un auditeur et que les versions qu'il a connues notamment à travers l'expérience de l'écoute se fixent dans sa mémoire même indépendamment de sa volonté.

Dans cette optique, la version italienne qui prime sur toutes les autres est Nell'acqua della chiara fontana, de Fabrizio De André, que nous avons découverte bien avant de connaître la chanson originale. Ainsi, le vers Ca la bbeddha stria me chiamau ('Que la jolie fille m'appelât') montre que, tout comme De André, nous donnons la parole « à la baigneuse, alors qu'elle était pure image dans la version française » (ABBRUGIATI, 2011: 18). Le choix du verbe vouloir au début des strophes 3 et 4, malgré les différences de temps et de personne, fait écho lui aussi à la version de De André, dont la troisième strophe commence justement par ce même verbe :

Volli coprire le sue spalle

'Je voulus couvrir ses épaules'

Par ailleurs, nous sommes redevable à Nanni Svampa car sa version en italien intitulée Nell'acqua del laghetto, a aussi influencé, de façon moins inconsciente, notre chanson d'arrivée. Tout d'abord, en choisissant un non mieux précisé laghetto ('petit lac'), au lieu de la claire fontaine, Svampa a situé la scène dans un paysage lacustre, un des lieux d'évasion favoris des habitants du nord de l'Italie. Ce qui nous a encouragée, à notre tour, à choisir comme décor un endroit situé dans le Salento. C'est aussi la version de Svampa qui nous a suggéré de terminer les vers 10 et 14 par une conjonction adversative : par une sorte de calque de l'italien au dialecte, nous avons adopté ma au lieu de però, ce qui a le double effet de créer un enjambement et de trouver une solution pour traduire de manière efficace un vers masculin.

\section{Conclusion}

Dans cette étude, nous avons illustré un cas de traduction chantée du français au dialecte du Salento, celui de la chanson Dans l'eau de la claire fontaine de Georges Brassens. À travers une analyse des stratégies adoptées pour recréer l'univers de Brassens dans une autre langue romane, nous avons contribué à mieux définir le critère de la chantabilité en traduction. En l'occurrence, nous avons montré que dans le cas d'une chanson en style syllabique dont les paroles présentent une régularité métrique, il est possible d'assimiler la notion de chantabilité au niveau métrique. 
Nous avons donc essayé de recréer, autant que possible, les structures métriques de la chanson originale: à l'inverse du mètre, qui n'a pas posé de problèmes particuliers, le schéma des cadences et des rimes n'est pas parfaitement recréé, notamment à cause de la difficulté à traduire les vers oxytons, une difficulté qui est peut-être encore plus grande dans le dialecte du Salento qu'en italien. Nous avons par ailleurs relevé que les versions de la même chanson dans d'autres langues jouent un rôle particulier dans le cas de la traduction chantée. Lorsqu'elles sont déjà bien connues par le traducteur, celui-ci les a tellement dans l'oreille qu'il n'arrive plus à savoir ce qui dérive de l'original et ce qui relève d'une traduction déjà existante.

En général, l'expérience personnelle de traduction nous a permis de réfléchir de manière plus approfondie aux enjeux de la traduction chantée. En effet, si la traduction est une affaire de trahison, une manière de «dire presque la même chose » (ECO, 2007), dans l'impossibilité de servir deux maîtres à la fois et avec le risque tangible de produire une belle infidèle, traduire une chanson est un véritable jeu d'équilibriste. Constamment suspendu entre les contraintes de la langue et celles de la musique, toujours en équilibre entre le sens et la forme, le traducteur de chansons est à la recherche continue d'un barycentre, et ses choix dépendent souvent de critères dont la subtilité est de l'ordre de la syllabe, voire de la voyelle. Et s'il n'a pas de talent inné, il devra apprendre à maîtriser les outils techniques nécessaires pour atteindre un résultat suffisamment agréable en termes de chantabilité.

\section{BIBLIOGRAPHIE}

AbBrugiati Perle (2011), Dans l'eau de la claire fontaine. Sur trois traductions de Brassens en italien, Cahiers d'études romanes 24, Université d'Aix-Marseille, p. 151-170. [En ligne], URL :

http://journals.openedition.org/etudesromanes/993.

ACHILle Elide (2007), Pour une analyse métrique des chansons de Brassens, Tesi di dottorato, Università di Bari, a.a. 2006-2007.

Beltrami Pietro G. (2002), Gli strumenti della poesia, Bologna, Il Mulino.

BRACOPS Martine (ed.) (1992), Traduire et interpréter Georges Brassens, Équivalences 22/1-2, 23/1, Bruxelles, ISTI.

BrasSEns Georges (1973), Poèmes et chansons, Paris, Éditions musicales 57.

CAMUgli Gallardo Catherine (2010), Jusqu'où la syntaxe construit-elle le sens ? Réflexions autour d'une comparaison italien-français des locutions verbales figées, Langages 179-180, Paris, Larousse, p. 243-258.

CONENNA Mirella (1987), Traduire la chanson : les interprétations italiennes de Georges Brassens, Le Français dans le Monde. Recherches et Applications, numéro spécial, Paris, CLE international/Hachette, p. 99-106.

ConennA Mirella (ed.) (1998), Georges Brassens. Lingua, poesia, interpretazioni, Fasano, Schena Editore.

CONENNA Mirella (2000), Dissolvenze incrociate : canzoni e traduzioni di Brassens, in : GARZONE Giuliana, ScHENA Leandro (ed.), Tradurre la canzone d'autore, Bologna, Clueb, p. 153-165. 
CORNULIER Benoît de (1999), Petit dictionnaire de métrique, polycopié, Université de Nantes. [En ligne], URL :

http://www.normalesup.org/ bdecornulier/DicoMet.pdf.

CORNULIER Benoît de (2004), Rime et contre-rime en tradition orale et littéraire, Poésie et chant, polycopié, Université de Nantes, p. 1-13. [En ligne], URL : https://www.normalesup.org/ bdecornulier/PoCh2.pdf.

D'ANDREA (2014), Stratégies métriques et traduction des textes chantés, in: WoJCIECHOWSKA Barbara (dir.), De la musique avant toute chose. Notes linguistiques et littéraires, Paris, L'Harmattan, p. 71-85.

D'ANDREA (2020), Les unités de traduction existent-elles dans la chanson traduite ?, Tradução em Revista 29, Rio de Janeiro, PUC-Rio, p. 42-66.

De ANGElis Enrico, Neri Michele, SetTIMo Franco (2017), Discografia, Vinile 10, p. $110-129$.

ECO Umberto (2007), Dire presque la même chose, Paris, Grasset.

GDLI : Salvatore Battaglia, Grande dizionario della lingua italiana, Torino, UTET, 1961-2002, 21 voll.

Grassi Corrado, Sobrero Alberto A., Telmon Tullio (1997), Fondamenti di dialettologia italiana, Bari, Laterza.

HIRSCHI Stéphane (2008), Chanson. L'art de fixer l'air du temps. De Béranger à Mano Solo, Paris, Les Belles Lettres/Presses Universitaires de Valenciennes.

LADMIRAL Jean-René (2015), Sourciers et ciblistes, in : Sourcier ou cibliste. Les profondeurs de la traduction, Paris, Les Belles Lettres, p. 3-27.

ROHLFS Gerhard (1966), Grammatica storica della lingua italiana e dei suoi dialetti. Fonetica, Torino, Giulio Einaudi Editore.

Svampa Nanni, MASCIOLI Mario (ed.) (1991), Brassens. Tutte le canzoni tradotte da Nanni Svampa e Mario Mascioli, Padoue, Franco Muzzio Editore.

VALLI Donato (ed.) (1998), Letteratura dialettale salentina. L'Ottocento, vol. 1, Galatina, Congedo editore.

ZopPI Sergio (1998), Brassens al Balon, in : ConENNA Mirella (ed.), Georges Brassens. Lingua, poesia, interpretazioni, Fasano, Schena Editore, p. 197-201. 